\title{
Caracterización de factores implicados en las conductas de riesgo en adolescentes ${ }^{1}$
}

\author{
Characterization of factors involved in risk behaviors in adolescents
}

Fabio Gabriel Salas ${ }^{2}$

Recibido: 27/06/2017. Corregido: 26/08/2017.

Aprobado: 4/04/2018.

\begin{abstract}
Resumen: Las conductas de riesgo en los adolescentes han cobrado gran relevancia en la actualidad. Esto se debe en parte a su impacto tanto en la vida de los jóvenes que las protagonizan, como en las consecuencias generales que estas conllevan para la comunidad. Surge así un interés científico por conocer los diversos factores asociados como una manera de comprender mejor estos comportamientos, aunque, muchos de estos estudios se sostienen en perspectivas limitadas que no consideran los factores que aquí se mencionan sobre esta problemática.
\end{abstract}

El presente trabajo pretende realizar una caracterización del concepto de conductas de riesgo que permita clarificar su importancia en la constitución del escenario donde tiene lugar el desarrollo de los adolescentes. Para ello se describen algunos de los componentes de mayor relevancia que configuran este tipo de comportamientos, así como, los factores que favorecen y obstaculizan su presencia.

Se concluye que cualquier propuesta para abordar este tipo de comportamientos debe tener en cuenta los diferentes aspectos implicados referidos en este trabajo, y el papel que cada uno de estos juega en la configuración de estas conductas.

Palabras claves: adolescencia; conflicto, inseguridad; conducta adolescente.

\begin{abstract}
Risk behaviors in adolescents have become highly relevant today. This is partially due to the impact it has on the lives of the young people involved, as well as the general consequences for the community. Consequently, a scientific interest to know the different factors involved has generated as a way to better understand these types of behavior, although many of these studies are supported by limited perspectives that do not consider the factors mentioned here.

This paper is aimed at characterizing the concept of risk behavior to clarify its importance in the adolescents' development. For such purpose, some of the most important components constituting these types of behavior are described, as well as the factors favoring and hindering their presence.

It is concluded that any proposal to analyze this type of behavior must take into account the different aspects involved mentioned here and the role each one plays in this behavior.
\end{abstract}

Keywords: adolescence; conflict, insecurity; teenage behavior.

1 Este trabajo se realizó en el marco del proyecto: La persona desde la perspectiva integradora. Estudio de la personalidad y promoción de salud (PROICO N4-1612), UNSL.

2 Doctor en Psicología, Profesor Adjunto Exclusivo en el área de Procesos Psicológicos Básicos del Departamento de Formación Básica General y Complementaria - Facultad de Psicología, Universidad Nacional de San Luis, Argentina. Correo electrónico: fgsalas@unsl.edu.ar. 


\section{Introducción}

Las conductas de riesgo representan un tema de importancia para diferentes áreas de conocimiento como la psicológica y la educativa, entre otras áreas sociales, donde se evidencia cada vez más interés en profundizar su estudio.

Este tipo de conductas se ha convertido en un problema que genera serias dificultades no solo a nivel personal, sino también, en el medio social inmediato de la persona: familia, ámbito educativo y laboral. Además repercuten en los recursos que, en particular, desde las áreas de salud, educación y justicia el Estado dirige a esta problemática; de sus disposiciones y posicionamientos al respecto deriva la implementación de estrategias dirigidas a su atención.

Para Celis y Vargas (2005, p. 108), la conducta riesgosa puede ser entendida como"toda conducta manifiesta o intra-verbal que atente contra el equilibrio o el desarrollo biológico, psicológico y social de la persona". Se consideran como conductas de riesgo: las conductas sexuales de riesgo, el abuso de alcohol, el consumo de tabaco, algunas conductas agresivas, el consumo de sustancias psicoactivas, entre otras. Estas se relacionan entre sí presentando factores de riesgo y de protección que les otorga la condición de comórbidas (Jane-Llopis \& Matytsina, 2006).

Este estudio se aborda con un enfoque metodológico mixto; se analizan varios trabajos con el objetivo de llamar la atención sobre la complejidad de las conductas de riesgo, las que se pueden evidenciar en elementos de distintas dimensiones que convergen en su surgimiento y desarrollo: por un lado factores individuales y grupales; y por el otro, factores psicológicos, sociales y culturales. De esta manera, se caracterizan los factores y aspectos más destacados implicados en el surgimiento y desarrollo de estos comportamientos.

\section{Las conductas de riesgo y los valores de la sociedad actual}

Las conductas de riesgo entendidas como esos comportamientos que pueden atentar contra el equilibrio y el desarrollo de una persona están presentes en los valores que priman actualmente en la sociedad.

En el presente contexto sociocultural se valora la actitud de asumir riesgos ante las decisiones que se tiene que tomar. El animarse y arriesgarse se suele utilizar para describir o valorar de manera positiva a las personas, resaltando así sus valentía y su determinación como rasgos que favorecen su personalidad en la vida social. En la cultura actual se destaca un sistema de valores donde el hecho de asumir riesgos tiene una connotación positiva y es valorado por la sociedad; y por medio de estas conductas las personas manifiestan otros valores que gozan del mismo prestigio: audacia, poder, iniciativa, entre otros. Esto conduce a que, ante distintas situaciones, se asuman riesgos sin medir totalmente la dimensión de las posibles consecuencias; esto es más frecuente en algunas etapas del desarrollo humano específicamente, en la adolescencia.

Uno de los motivos que favorecen la prevalencia de los comportamientos de riesgo es que muchos de sus aspectos son desconocidos o aún no han sido estudiados con profundidad, 
esto exige investigaciones que conduzcan a políticas y programas de prevención para disminuir estas conductas y sus consecuencias (Rosabal García, 2015); y de este modo ayudar a los jóvenes a enfrentar estos posibles riesgos pensando no solo en causas individuales o del medio social inmediato, sino también considerando el contexto macro-social en el que emergen y se desarrollan.

La presencia de conductas de riesgo en los adolescentes los expone a diferentes peligros que pueden dañar su salud y ocasionar consecuencias que perjudiquen su futuro; los grupos de jóvenes que realizan alguno de estos comportamientos estarían más propensos a llevar cabo otros tipos de conductas riesgosas, lo que los deja en un estado de mayor vulnerabilidad frente a la posibilidad de vincularse con personas y lugares que implican un daño potencial para su integridad (Florenzano Urzúa y Correa Valdez, 2005).

La manera en que se buscan experimentar muchas situaciones de la vida social, y el significado que las personas le otorgan a estas experiencias, ha originado lo que Donas Burak (2001) ha denominado "cultura de riesgo"; donde ciertos valores se vinculan entre sí bajo un mismo modelo de vida, que de manera excesiva tiende a cultivarse especialmente entre los jóvenes, y que promueve conductas de riesgo e incluso algunas patologías (Paramo, 2011). Muchas conductas de riesgo están relacionadas con el surgimiento de diferentes enfermedades en la edad adulta, y estas a su vez se relacionan con otros factores como la menor esperanza de vida, la calidad de vida, los gastos en la salud de la población y con el desarrollo económico y social de las comunidades (Restrepo Henao, Duque Ramírez y Montoya Gómez, 2012).

Se puede decir que estas conductas tienen sus raíces en un determinado estilo de vida, que contiene una serie de valores, lógica y dinámica que posibilitan su expresión y reconocimiento. El estilo de vida se concibe como "la manera particular en que cada individuo se relaciona consigo mismo y con su medio ambiente" (Celis y Vargas 2005, p. 108). En este caso, el estilo de vida más valorado, se sostiene a partir de la vigencia de preceptos sociales y culturales actualmente globalizados como la búsqueda de nuevas experiencias y con ellas nuevas sensaciones, el desafío de los límites y la competitividad entre las personas. Esto ha provocado que se naturalicen y se promocionen comportamientos y conductas de riesgo que hace algunas décadas atrás no tenían la prevalencia que hoy tienen en la vida social. De acuerdo a Oliva (2004) se estaría en presencia de cierta ambivalencia frente al tratamiento social que reciben estas conductas; ya que si bien, por un lado, se han definido sus límites de manera precisa, por otro lado, la cultura empuja e impele a asumir nuevos peligros y retos, nuevas experiencias, provocando el cruce de la línea divisoria hacia conductas inseguras; las mismas que, en la etapa de la adolescencia, encuentran su razón en la necesidad de experimentación, de implementar conductas de exploración y de asumir peligros en pos de alcanzar autonomía y la construcción de una identidad; es decir que se asumen conductas de riesgo como una oportunidad para el desarrollo y el crecimiento personal.

El comportamiento en las interacciones sociales se ha complejizado y su estudio requiere de perspectivas que puedan dar cuenta de estos cambios. Los sistemas de referencia que ayudaban a comprenderlas se han ido transformando con los cambios culturales y socioeconómicos de 
las últimas décadas promoviendo nuevas referencias para las decisiones más importantes en la vida de las personas; lo cual requiere ampliar la perspectiva de análisis para cualquier estudio social que se quiera realizar (Agudo Arroyo, 2004). Como expresan Vega, Aramendi y Garín (2012), ante los comportamientos que implican cierto peligro, hay que considerar que:

Las conductas de riesgo exigen una lectura social para ir más allá de sus manifestaciones más próximas y ofertar respuestas a corto y a largo plazo más coherentes con sus necesidades, tareas que parecen quedar marginadas en una sociedad más pendiente de los beneficios del mercado que de los derechos de las personas (p. 171).

Estos mismos autores entienden que estas conductas deben ser analizadas teniendo en cuenta las características de su contexto de surgimiento, ya que ellas son un reflejo de la sociedad en la que tienen lugar.

La relación entre el riesgo que asumen hoy las personas y la sociedad de consumo, ofrece un panorama esclarecedor sobre este nexo y el reforzamiento recíproco existente entre ambos. El triunfo del individualismo moderno como forma de existencia en la actualidad implica la elección sobre diferentes aspectos de la vida personal por fuera del marco de sujeto social antes dominante, como un individuo en condición de consumidor global; donde el riesgo representa un nuevo campo de acción convirtiéndose en un elemento que condiciona tanto los rasgos personales como el de los grupos sociales (Arenas Berrío, 2012).

\section{Conductas de riesgo en adolescentes}

El desarrollo del adolescente puede verse alterado por acciones voluntarias del propio joven entre las que se encuentran las conductas o comportamiento riesgosos, que pueden llevar a consecuencias nocivas para su salud. Estas conductas tienden a no distribuirse en forma aleatoria entre todos los/as adolescentes de una población dada, sino a concentrarse en un conjunto de estos, que habitualmente presentan algunas de estas conductas de riesgo. En muchos casos distintos tipos de conductas de riego se asocian entre sí: el consumir drogas, tener actividad sexual precoz, presentar conductas sexuales particulares y tener bajo rendimiento escolar suelen ser comportamientos de un mismo grupo de adolescentes (Florenzano Urzúa y Correa Valdés, 2005).

Teniendo en cuenta los cambios que se experimentan en la adolescencia, existe la posibilidad de que la adaptación del joven -y por ende de su familia- al medio social, no se realice de forma armoniosa, lo que implica el peligro de que surjan conductas de riesgo que pueden llegar a dificultar su desarrollo presente y futuro (Fernández, 2005). Al respecto, se sostiene que:

La adolescencia se caracteriza por ser clave en la adquisición de los estilos de vida, saludables o problemáticos. La adquisición de un mayor nivel de independencia del ambiente familiar y un mayor compromiso con el grupo de iguales puede llevar a los adolescentes a nuevas situaciones donde se enfrentan a la toma de decisiones que pueden afectar su futuro; en esta etapa los jóvenes pueden incurrir en conductas 
problema como fumar, beber, uso de drogas, iniciar su vida sexual, tener relaciones sexuales desprotegidas o bajo el influjo de alcohol o drogas, manejar a exceso de velocidad o bajo el influjo del alcohol (Díaz Rodríguez y González Ramírez, 2014, p. 2).

Las conductas de riesgo se conciben a partir de las implicancias o consecuencias que las mismas pueden tener en la salud del adolescente, "Es así que se define como conducta de riesgo a cualquier comportamiento que comprometa los aspectos biopsicosociales del desarrollo exitoso del adolescente" (Sánchez, 2013, p. 30). Estas conductas no solo afectan notablemente la salud y la vida del adolescente, sino que también pueden condicionar sus posibilidades de ser afectados por enfermedades crónicas no trasmisibles en su adultez y de tener una inserción social exitosa (Barber \& Schluterman, 2008).

En este sentido, los importantes cambios socioculturales de diversos países como los procesos de transición demográfica de las últimas décadas, requieren de una revisión de las consideraciones sobre la salud y la enfermedad en los adolescentes. Actualmente muchos de sus problemas de salud derivan de conductas de riesgo que traen como consecuencias distintas afecciones, como por ejemplo, enfermedades de transmisión sexual, accidentes de tránsito, depresión y suicidio, entre otros perjuicios (Blum \& Nelson-Mmari, 2004).

En la adolescencia las conductas de riesgo pueden ser determinantes de la salud, siendo causales de morbilidad y de mortalidad en esta etapa. Algunas de las conductas de riesgo más importantes que se presentan en la adolescencia son el consumo de drogas, la mala alimentación, la depresión y el suicidio, las conductas que conducen a la adquisición de infecciones de transmisión sexual (ITS) el embarazo precoz; como así también, la ingesta de alcohol, el sedentarismo y la producción de lesiones no intencionales (Roche, Ahmed \& Blum, 2008; Breinbauer \& Maddaleno, 2008; Sánchez, 2013). Según Intra, Roales-Nieto y San Pedro, (2011) estos riesgos también pueden resumirse mencionando que la etapa de la juventud principalmente se caracteriza por la vulnerabilidad ante la posibilidad de iniciarse en el tabaquismo, la drogadicción y las prácticas sexuales inseguras.

También hay que considerar que el riesgo, en cierta medida, es parte de las conductas propias del desarrollo en la etapa de la adolescencia, siendo necesaria y saludable una cuota del mismo en sus decisiones y comportamientos. Los jóvenes que no asumen peligros de ningún tipo pueden presentar dificultades en distintos aspectos de su desarrollo. En relación a esta idea se advierte que:

Es posible que una actitud adolescente conservadora y de evitación de riesgos esté asociada a una menor incidencia de algunos problemas comportamentales y de salud, sin embargo, también es bastante probable que esa actitud tan precavida conlleve un desarrollo deficitario en algunas áreas, como el logro de la identidad personal, la creatividad, la iniciativa personal, la tolerancia ante el estrés o las estrategias de afrontamiento (Oliva, 2004, p. 119). 


\section{El contexto social como un agente principal}

El estudio de estos comportamientos se puede llevar a cabo desde distintos enfoques. Pero según Gil García y Romo Avilés (2008), para poder comprender el hecho de que una persona asuma ciertos peligros es necesario un cambio de unidad de análisis del individuo a las relaciones e interacciones sociales, ya que estas conductas no son producto de decisiones individuales sino más bien de un proceso social.

Desde esta perspectiva, se plantean diferentes análisis donde las razones de las conductas de riesgo se encuentran en la interacción social en distintos niveles entre el adolescente y su contexto social: desde la relación que mantiene con los integrantes de su familia y con el grupo de amigos, pasando por los vínculos que establece en el colegio, el barrio y la comunidad; hasta la valoración que le otorga a los distintos agentes que, de manera masiva, tienen la capacidad de influir y condicionar sus conductas.

Las primeras redes sociales están constituidas por la familia, amigos, vecinos, compañeros de la escuela y conocidos de la comunidad; estos se convierten en un sistema de apoyo social que resulta indispensable para la salud, el ajuste y el bienestar del joven, es decir, que estas redes sociales constituidas por personas significativas para la persona, son fundamentales (Orcasita y Uribe, 2012). En otras palabras "en el desarrollo de un adolescente, el contexto social en que se desenvuelve -familia y escuela- son los contextos más influyentes" (Santander et al., 2008, p. 318).

Existen otros niveles de interacciones donde intervienen factores que influyen en las experiencias que van teniendo los jóvenes al atravesar la etapa de la adolescencia. Le Breton (2003), señala que la dificultad para el pasaje a la vida adulta se ve acentuada por la confusión de los referentes de sentido y de valores, que caracteriza a nuestras sociedades contemporáneas. Actualmente los jóvenes sienten una fuerte presión por sentirse parte de la sociedad, y por encontrar un lugar que satisfaga sus necesidades psicosociales. Pero el contexto social no siempre le responde de manera favorable. En este sentido, se advierte que:

Se trata de un proceso condicionado desde las instituciones sociales que obligan a los jóvenes a desarrollar su propia biografía y su vida individual, al depender del mercado estudiantil, laboral, de las condiciones de vida, del consumo, de las regulaciones sociales (Velásquez, 2009, p. 37).

Asimismo es necesario atender a las características socioeconómicas del contexto donde se encuentra el adolescente; la falta de recursos sociales y materiales puede configurar escenarios que conduzcan a diferentes conductas de riesgo. Las crisis económicas y sociales que vive la sociedad impacta en el colectivo de adolescentes y jóvenes, y si no se da respuesta a esas necesidades se terminan reforzando esos problemas y al mismo tiempo se coartan sus posibilidades de desarrollo personal en el futuro, reduciéndolo a sufrir algún tipo de exclusión social (Vega et al., 2012). Los bajos recursos económicos y sociales de una familia pueden estar asociados a las conductas de riesgo de los hijos: estas familias tienden a residir en zonas donde 
la violencia y el consumo de drogas es más frecuente que en otros lugares, lo cual favorece la trasmisión de estos hábitos por medio de los pares y del barrio o la comunidad (Cardoso y Verner, 2007).

Los jóvenes que están implicados en conductas de riesgo requieren una atención coherente a sus necesidades, de otra manera se corre el peligro de excluirlos, reducir sus derechos y afectar aún más su futuro. La sociedad actual, en estado de permanente crisis, está dificultando la inserción social de todos los jóvenes, pero más aún, de los que provienen de sectores de pobreza y marginalidad donde se pueden generar más fácilmente este tipo de conductas. Vega et al. (2012) resumen este pensamiento:

Los problemas de los adolescentes y jóvenes nunca están al margen de la sociedad en la que viven. Por esto al analizar sus conductas de riesgo resulta imprescindible contextualizar sus vidas al mismo tiempo que comprender su proceso vital. Sus conductas son un reflejo de la sociedad en la que viven (p. 168).

\section{Un análisis desde la toma de decisiones}

Desde otra perspectiva, se analiza el comportamiento adolescente en función de los procesos racionales vinculados a la toma de decisiones. Cueto, Saldarriaga y Muñoz (2011), describen las posibles causas que conducen al tipo de conductas mencionando que, desde la economía y la psicología, se pueden identificar tres enfoques que describen los mecanismos de inicio y mantención de las conductas de riesgo: el enfoque de la economía clásica; el enfoque de la psicología del desarrollo; y el enfoque de la economía del comportamiento. Los mismos se distinguen a partir de las características concretas que diferencian la toma de decisiones (entendida como determinante de conductas de riesgo) de adultos y adolescentes; y en el tipo de incentivo propuesto en la explicación de estas conductas.

En el primer enfoque, de la economía clásica, las decisiones tomadas en relación a realizar alguna conducta de riesgo estarían dadas a partir de cuestionarse si los beneficios de llevar a cabo una conducta de este tipo excede o no los costos asociados a dicha actividad. En situaciones de incertidumbre de los costos y beneficios en juego, se toma la decisión con base en la comparación de las utilidades esperadas. Hasta aquí no habría diferencia entre adolescentes y adultos, pero la misma se puede presentar en los incentivos que para cada uno se ponen en juego para incurrir en actividades de riesgo.

En el segundo enfoque de la psicología del desarrollo, la variedad de factores que podría influir en las decisiones del adolescente para incurrir en conductas de riesgo es mucho más amplia. En este caso se consideran los niveles de desarrollo que el adolescente ha alcanzado en diferentes órdenes: desarrollo cognitivo (qué piensa acerca del mundo), desarrollo afectivo (qué sienten las personas acerca del mundo) y desarrollo social (el rol que otras personas juegan en la determinación de las elecciones de los individuos). Con base en estas dimensiones del desarrollo se pueden encontrar diferencias y similitudes entre el adolescente y el adulto ante determinadas situaciones que implican asumir un riesgo. 
En el tercer enfoque de la economía del comportamiento se incorporan otras dimensiones estudiadas en la psicología del desarrollo respecto a la toma de decisiones en los adolescentes. Así, por ejemplo O'donogue y Rabin (2000), consideran que existen dimensiones donde se pueden identificar los factores que explican las conductas de riesgo en los jóvenes: la dimensión de las preferencias intertemporales (comparado con los adultos, los adolescentes pueden estar subestimando las consecuencias de sus actos), la dimensión de los beneficios futuros (equivocarse respecto a cómo sentirán en el porvenir con las consecuencias de sus decisiones actuales) y la dimensión del número de ocasiones que el individuo se encuentra en la necesidad de optar por incurrir en una conducta de riesgo.

\section{Factores de riesgo y factores protectores}

Teniendo en cuenta la situación de vulnerabilidad y la condición de dependencia en la etapa de desarrollo evolutivo que atraviesa el adolescente, diferentes situaciones de su medio social pueden convertirse en condicionantes que promuevan el surgimiento de determinadas conductas peligrosas. En cada contexto social y cultural se pueden identificar distintos factores, tanto protectores como de riesgo, que juegan un papel central en la presencia de estos comportamientos.

En este sentido, en los últimos años ha cobrado importancia el hecho de conocer los factores implicados en las conductas inseguras, y de esta manera, buscar su prevención y su mejor tratamiento (Florenzano Urzúa y Correa Valdés, 2005).

\section{Factores de riesgo}

Pineda Pérez y Aliño Santiago (1999), definen los factores de riesgo como los elementos que pueden tener para el adolescente un alto nivel de probabilidades de resultados no deseados 0 de daño, por lo que requieren de intervención. Dentro de este enfoque se han podido identificar importantes factores, que aunque no constituyen en sí conductas de riesgo de adolescentes, han sido señalados como eslabones de cierto peligro que incrementan la vulnerabilidad juvenil, y que están presentes en los contextos o medios sociales donde el individuo se desenvuelve y en sus antecedentes de personalidad. De esta manera se puede expresar, a modo de definición, que "los factores de riesgo son el escenario donde se desarrollan las conductas problema o conductas de riesgo" (Díaz Rodríguez y González Ramírez, 2014, p. 2).

Los factores de riesgo muchas veces están representados por ciertas conductas peligrosas ya existentes en los hábitos del adolescente; es decir, que generalmente se superpone la presencia de conductas peligrosas llevadas a cabo en determinados momentos del desarrollo, con la función que estas adquieren como factores causales de riesgo en futuras conductas de este tipo.

Distintos comportamientos, situaciones y condiciones han sido identificados como factores de riesgo para la salud. Los mismos funcionan como tal según ciertas condiciones como son la edad, el contexto, la época, entre otras, de los jóvenes a los que se hace referencia. 
Al respecto, se han realizado investigaciones que identifican posibles factores de riesgo en los adolescentes, donde se advierte que la validez de los mismos está limitada a explicar los comportamientos de riesgo del grupo de jóvenes que constituye la muestra del estudio. Si bien estos factores no son generalizables, permiten dar cuenta de cuáles serían los aspectos a tener en cuenta cuando se hace referencia a estos condicionantes.

Para Molinatti y Peláez (2012), según trabajos realizados en la ciudad de Córdoba, Argentina, las condiciones socio-habitacionales desfavorables y el clima educativo bajo en el hogar, pueden conducir a que los jóvenes tengan mayores probabilidades de manifestar comportamientos de riesgo. En otro estudio sobre adolescentes de la ciudad de Calama, Chile se observó que las variables más destacadas en este sentido son la falta de destrezas sociales, ausencia de grupo de pertenencia, bajo autoconcepto/autoestima y situaciones familiares irregulares (Haquin, Larraguibel y Cabezas, 2004).

Asimismo, Paniagua Repetto y García Calatayud (2003), han observado otros factores de riesgo en episodios de embriaguez, mayor consumo de tabaco, alcohol y drogas ilegales, conductas violentas, mayor consumo de sustancias adictivas por amigos y familiares y trastornos del aprendizaje. Del mismo modo, Barcelata, Durán y Lucio (2004), entienden que algunos factores de riesgo pueden estar representados en la tendencia al aislamiento, preocupación por la autoimagen, problemas emocionales en distintas áreas, en particular en lo familiar y en la salud.

Por otro lado, los factores de riesgo también son estudiados desde abordajes que consideran distintos niveles de la vida social. En un nivel macro social se estima que las condiciones socioeconómicas desfavorables y el bajo nivel sociocultural, pueden provocar consecuencias como: insuficiente rendimiento escolar, poco desarrollo de habilidades básicas y baja resistencia a la influencia de los pares, entre otras cosas; favoreciendo las conductas de riesgo en la población adolescente (Sánchez, 2013).

Siguiendo con la idea de diferenciar los factores causales de estos comportamientos en función de distintos niveles, Donas Burak (2001), menciona que existirían factores de riesgo de "amplio espectro" y los factores o conductas que pueden funcionar de manera específica para un tipo de daño. Entre los factores de amplio espectro se puede mencionar: el pertenecer a un grupo con conductas de riesgo, la deserción escolar, el no tener un proyecto de vida relativamente definido, familia con vínculos deteriorados, violencia familiar, tener baja autoestima y bajo nivel de resiliencia. En relación a este grupo de factores Santander et al. (2008), expresan que en adolescentes provenientes de familias percibidas por ellos como disfuncionales, todas las conductas de riesgo son más frecuentes, lo que indica la necesidad de implementar estrategias para fortalecer la familia, teniendo en cuenta la importancia de la misma como factor de prevención de estas conductas. En este sentido, Hernández-Castillo, Cargill-Foster y Gutiérrez-Hernández (2012), sostienen que hay que tener en cuenta que los cambios en la estructura familiar como el ingreso de la mujer al mundo laboral y el incremento de las familias monoparentales, han provocado mayor vulnerabilidad en estas estructuras sociales que antes funcionaban como un elemento protector para los jóvenes. 
Dentro del posible nivel de alcance o repercusión de los factores riesgosos, los que de manera específica pueden predisponer a ciertos comportamientos de riesgo serían: consumir alcohol, depresión, portar armas y conducir sin protección (Donas Burak, 2001). Del mismo modo, algunas estrategias de afrontamiento (estilo no productivo) como estrategias de evitación y de presión del grupo, estarían funcionando como factores de riesgo para algunas conductas como el inicio de consumo de drogas y la implicación en actos antisociales (Gómez Fraguela, LuengoMartín, Romero-Triñanes, Villar-Torres y Sobral-Fernández, 2006).

\section{Factores protectores}

Así como se han identificado factores de riesgo de las conductas peligrosas en los adolescentes, también existen otras situaciones, características o condiciones del medio social, atributos o cualidades personales que funcionan como factores protectores para las mismas. Los estudios internacionales indican que en la vida de las personas adolescentes existen factores protectores que son los que les permitirían desarrollarse adecuadamente llegando a la adultez con cierto éxito y siendo un personas responsables (Kosttelecky, 2005).

La salud de las adolescentes y los adolescentes está condicionada a la presencia de estos factores de protección. Se ha señalado al respecto que:

En el campo de la salud, hablar de factores protectores, es hablar de características detectables en un individuo, familia, grupo o comunidad que favorecen el desarrollo humano, el mantenimiento o la recuperación de la salud; y que pueden contrarrestar los posibles efectos de los factores de riesgo, de las conductas de riesgo y, por lo tanto, reducir la vulnerabilidad, ya sea general o específica (Páramo, 2011, p. 87).

Al analizar los factores protectores según la manera en la que influyen o condicionan la presencia de conductas de riesgo, Donas Burak (2001) propone dos tipos de factores: de amplio espectro (con mayor capacidad indicativa sobre la posibilidad de que el adolescente tenga estas conductas), y factores protectores específicos capaces de indicar el posible surgimiento de un grupo determinado de estos comportamientos.

Entre el primer grupo de factores se encuentran: permanecer en el sistema educativo formal, un alto nivel de resiliencia, locus de control interno bien establecido, sentido de la vida elaborado, alta autoestima; la construcción de un proyecto de vida y familia contenedora con buena comunicación interpersonal. Dentro de este tipo de factores, Blum, et al., (2003), mencionan que un apoyo fundamental para el adolescente es el contexto que configuran la familia, la escuela y las amistades; los mismos están señalados en la literatura especializada como factores protectores de gran importancia, aunque en ciertos casos, también pueden funcionar como factores de riesgo.

Al respecto, es necesario señalar que si bien el seno familiar ha sido considerado en muchas ocasiones un marco de resguardo para un adolescente, ya en el siglo XX otras instituciones como la educativa, comenzaron a tener mayor peso en la vida de esas personas. Para Gonzales 
y Yedra (2006) la escuela, y en particular el interés que los profesores muestren por los jóvenes a través de consejos y orientación, son factores protectores para evitar el consumo de sustancias adictivas. En las últimas décadas, los fuertes cambios (las nuevas tecnologías, los medios de comunicación utilizados y la globalización), llevaron a que el valor de cualquier elemento considerado como factor protector sea más relativo, y al mismo tiempo, a que el adolescente se encuentre expuesto a una mayor cantidad de factores que influyen en su desarrollo.

Dentro de los factores protectores específicos que pueden evitar ciertas conductas de riesgo o el daño en la salud del adolescente, según Donas Burak (2001) se encontrarían por ejemplo: mantener relaciones sexuales con los cuidados adecuados que permitan controlar el embarazo y las infecciones por transmisión sexual, la abstención en el uso del cigarro y el uso de medidas de seguridad en la conducción de vehículos. Como factores protectores específicos también se pueden mencionar algunas estrategias de afrontamiento propias de los estilos de afrontamiento productivo: esforzarse y concentrarse en resolver los problemas y fijarse en lo positivo. Estas parecen actuar como protección ante la implicación en actos antisociales y el inicio de consumo de drogas (Gómez Fraguela et al., 2006). Al mismo tiempo, estos estilos de afrontamiento productivos están asociados a altos niveles de bienestar psicológico en los jóvenes (Figueroa, Cohen Imach de Parolo, Lazunca y Gronda, 2005).

Por otrolado, Florenzano Urzúa y Correa Valdez(2005), sostienen que distintos estudios empíricos concluyen que algunos de los factores protectores más importantes son: una relación constante con los padres, relaciones sociales estables dentro y fuera de la familia, modelos sociales que valores el enfrentamiento positivo de los problemas, experiencias de autoeficacia, con locus de control interno, balance adecuado entre responsabilidades sociales y expectativas de logro, actitud proactiva frente a situaciones estresantes y una experiencia de sentido y significado de la propia vida.

\section{Análisis final}

Los comportamientos de riesgo que llevan a cabo los adolescentes entre los que se puede mencionar principalmente el alcoholismo, la drogadicción, el tabaquismo y las conductas sexuales inseguras, la mala alimentación, la depresión y el suicidio, el sedentarismo y la producción de lesiones no intencionales, presentan distintos procesos involucrados. Se pueden analizar estos procesos como elementos de tres componentes más generales que rigen el desarrollo adolescente actualmente: los procesos evolutivos propios de la etapa adolescente, la preminencia de valores culturales que promueven actitudes que conducen a asumir riesgos en muchas de las decisiones que se toman, y el tipo de influencia que recibe el adolescente de distintos niveles de su contexto social (la familia y el grupo de pares; los vínculos que establece en el colegio, el barrio y la comunidad y el impacto de agentes sociales, como los medios de comunicación, que de manera masiva influyen y condicionan sus conductas). El intento por poder identificar el nivel de implicancia de los agentes asociados al surgimiento y desarrollo de las conductas de riesgo no es fácil, ya que como cualquier comportamiento humano está atravesado por una serie de condicionantes que no solo actúan de manera simultánea en un determinado momento evolutivo, sino que muchos de ellos acompañan los procesos de 
cambio en cada joven. Los factores promotores de las conductas de riesgo pueden llegar a presentarse y promover intensos procesos de interacción con el medio social que lo rodea, los que conducen a establecer nuevas condiciones para su desarrollo.

En la adultez e incluso en algún grupo de jóvenes de la llamada "juventud tardía", estos comportamientos encuentran condiciones menos favorables para su surgimiento y desarrollo. Pero en cambio la adolescencia, es un periodo del desarrollo en el que estos comportamientos pueden alcanzar altos niveles de prevalencia; la consolidación de una identidad a partir de los procesos de búsqueda de autonomía e independencia y del reconocimiento social de su entorno social, dan lugar a la necesidad de asumir ciertas situaciones de alto riesgo sin estar preparados para enfrentarlas de manera conveniente. No es extraño entonces que la atención de científicos, especialistas y profesionales se haya centrado en jóvenes de esta edad y en su condición de vulnerabilidad que los conduce a llevar a cabo acciones que comprometen su bienestar.

Estas conductas se convierten en un hecho que atraviesa al conjunto de la sociedad, ya que no solo puede resultar afectada la salud y el desarrollo del adolescente sino también la salud de la comunidad y su bienestar general; sus repercusiones alcanzan el ámbito sanitario, educativo y jurídico.

La adolescencia no es el único factor relevante para el desarrollo de las conductas de riesgo, la llamada sociedad o cultura del riesgo también juega un papel destacado. Actualmente existe una dinámica sociocultural que promueve este tipo de comportamientos; se invita continuamente a las personas a que se animen a probar, a arriesgar, a conocer lo nuevo y sentir la sensación del peligro. Esta actitud es valorada socialmente y se ha convertido en un medio para ganar reconocimiento ante los demás y como parte de un estilo de vida. Es lógico que los adolescentes, que por las razones antes mencionadas están más predispuestos a inclinarse por seguir estos mandatos, terminen asumiendo riesgos o peligros excesivos que muchas veces pueden tener consecuencias negativas para sí mismos o para los demás.

Si bien los estudios sobre el tema tienen a abordarlo desde una perspectiva reduccionista que destaca solo uno o algunos factores, el avance hacia una mayor comprensión de las conductas de riesgo requiere una mirada amplia donde estén incluidos los diferentes agentes que, en mayor o en menor medida, contribuyen a su desarrollo.

Hay pocos estudios que se hayan propuesto abordar el problema de las conductas de riesgo en los adolescentes de manera integral o al menos tratando de incluir elementos comunales, sociales y culturales; esto se debe especialmente a que su estudio presenta muchas dificultades y requieren de grandes esfuerzos y tiempo. Sin embargo, contar con un mayor desarrollo en este tipo de estudios permitiría obtener resultados de mayor alcance y envergadura, donde el análisis de distintos factores y la relación entre estos permitan alcanzar conclusiones más ajustadas a la dinámica de estos comportamientos. 


\section{Referencias bibliográficas}

Agudo, Y. (2004). Comas, D.(coord.),. Aguinaga, J.,. Orizo, F., Espinosa A. y E. Ochaita. Jóvenes y estilos de vida. Valores y riesgos en los jóvenes urbanos. Madrid: FAD, INJUVE, (2003). Critica de libro. Revista Española de Sociología, 4, 307-310. Recuperado de: http://www.fes-sociologia.com/ files/res/4/17.pdf.

Arenas, J. A. (2012, 19 de agosto). La sociedad de consumidores y de riesgo social. Sociedad Cronopio 33. Recuperado de: http://www.revistacronopio.com/?p=8547.

Barber, B. y Schluterman, J. (2008). En Valenzuela M. T., Ibarra R., Zubarew G. y Loreto Correa M. (2013). Prevención de conductas de riesgo en el Adolescente: rol de familia. Index de Enfermería, 22(1-2), 50-54. http://scielo.isciii.es/scielo.php?script=sci_arttext\&pid=S1132-1296201300010001.

Barcelata, B., Durán, C. y Lucio, E. (2004). Indicadores de malestar psicológico en un grupo de adolescentes mexicanos. Revista Colombiana de Psicología, 13, 64-73. Recuperado de: https:// revistas.unal.edu.co/index.php/psicologia/article/view/1210/1762.

Blum, R. W., Halcón, L., Beuhring, T., Pate, E., Campell-Forrester, S., \& Venema, A. (2003). Adolescent health in the Caribbean: risk and protective factors. American Journal of Public Health, 93(3), 456-460. Recuperado de: http://ajph.aphapublications.org/doi/abs/10.2105/AJPH.93.3.456.

Blum, R. W., \& Nelson-Mmari, K. (2004). The health of young people in a global context. Journal of Adolescent Health, 35(5), 402-418. https://doi.org/10.1016/j.jadohealth.2003.10.007.

Breinbauer, C. y Maddaleno, M. (2008). El modelo "Jóvenes: opciones y cambios" y su aplicación en el diseño de intervenciones eficaces para los adolescentes. En Organización Panamericana de la Salud (Eds.) Jóvenes: Opciones y Cambios. Promoción de Conductas Saludables en los Adolescentes. (pp. 33- 38). Washington DC: OPS. Recuperado de: http://publications.paho. org/spanish/PC+594+cap_7.pdf.

Cardozo, A. R. y Verner, D. (2007). En S. Cueto, V. Saldarriaga y I G. Muñoz, (2011). Conductas de riesgo entre adolescentes peruanos: un enfoque longitudinal. http://biblioteca.clacso.edu.ar/Peru/ grade/20120209054026/ddt58.pdf

Celis, R. y Vargas, C. (2005). Estilo de vida y conductas de riesgo. Sinéctica, 25, 108-113. Recuperado de: https://sinectica.iteso.mx/index.php/SINECTICA/article/view/276/269.

Cueto, S., Saldarriaga, V. y Muñoz, I., (2011). Conductas de riesgo entre adolescentes peruanos: un enfoque longitudinal. Consejo Latinoamericano de Ciencias Sociales (CLASCO) (Ed.) (pp. 118-165). Lima, Perú: GRADE. Recuperado de: http://biblioteca.clacso.edu.ar/Peru/ grade/20120223052104/30_cueto_saldarriaga_munoz. 
Díaz, C. L. y González Ramírez, M. T. (2014). Conductas problema en adolescentes en la ciudad de Monterrey, México. Enfermería Global, 13(33), 1-16. Recuperado de: http://revistas.um.es/ eglobal/article/view/184861/156691.

Donas, S. (2001). Protección, riesgo y vulnerabilidad: Sus posibles aplicaciones en la promoción, prevención, tratamientoy rehabilitación de la saludintegral delos adolescentesy las adolescentes. En S. Donas Burak. (ed.) Adolescencia y juventud en América Latina (pp. 489-499). Costa Rica, Cartago: Libro Universitario Regional.

Fernández, M. (2005). Relaciones familiares y ajuste en la adolescencia. Disertación doctoral no publicada, Universidad de Valencia, España. Recuperado de: https://www.uv.es/lisis/otraspublica/tesis_manuela.pdf.

Figueroa, M.I., Cohen Imach de Parolo, S., Betina Lacunza, A., Gronda, M. N., Amaya, A., y Brunella Block, A. (2005). Análisis de situaciones problemáticas autopercibidas por adolescentes (Tucumán, Argentina). Revista lberoamericana de Diagnóstico y Evaluación-e Avaliação Psicológica, 1(19). Recuperado de: http://www.redalyc.org/html/4596/459645450006/.

Florenzano Urzúa, R. y Correa Valdez, M. (2005). El adolescente y sus conductas de riesgo. ( $3^{\circ}$ Ed.) Santiago, Chile: Ediciones Universidad Católica de Chile.

Gil García, E. y Romo Avilés, N. (2008). Conductas de riesgo en adolescentes urbanos andaluces. Misceláneas Comillas, 66(129), 493-509. Recuperado de: http://revistas.upcomillas.es/index. php/miscelaneacomillas/article/view/7379/7225.

Gómez-Fraguela, J. A., Luengo-Martín, A., Romero-Triñanes, E., Villar-Torres, P. y Sobral-Fernández, J. (2006). Estrategias de afrontamiento en el inicio de la adolescencia y su relación con el consumo de drogas y la conducta problemática. International Journal of Clinical and Health Psychology, 6(3), 581-597. Recuperado de:http://www.aepc.es/ijchp/articulos_pdf/ijchp-193. pdf.

González, M. y Rey Yedra, L. R. (2006). La escuela y los amigos: Factores que pueden proteger a los adolescentes del uso de sustancias adictivas. Enseñanza e Investigación en Psicología, 11(1), 23-37. Recuperado de: http://www.redalyc.org/pdf/292/29211102.pdf.

Haquin, C., Larraguibel, M., y Cabezas, J. (2004). Factores protectores y de riesgo en salud mental en niños y adolescentes de la ciudad de Calama. Revista chilena pediatría, 75(5), 425-433. http://dx.doi.org/10.4067/S0370-41062004000500003.

Hernández-Castillo, L., Cargill-Foster, N. R. y Gutiérrez-Hernández, G. (2012). Funcionalidad familiar y conducta de riesgo en estudiantes de nivel medio superior Jonuta, Tabasco 2011. Salud en Tabasco, 18(1), 14-23. Recuperado de: http://salud.tabasco.gob.mx/sites/all/files/sites/salud. tabasco.gob.mx/fi/Vol18,\%20num1_completo_0.pdf\#page=19. 
Intra, M. V., Roales-Nieto, J. G. y San Pedro, E. M. (2011). Cambio en las conductas de riesgo y salud en estudiantes universitarios argentinos a lo largo del periodo educativo. International Journal of Psychology and Psychological Therapy, 11(1), 139-147. Recuperado de: http:// www.ijpsy. com/volumen11/num1/289/cambio-en-las-conductas-de-riesgo-ysalud-ES.pdf.

Jane-Llopis, E. \& Matytsina, I. (2006). Mental health and alcohol, drugs and tobacco: a review of the comorbidity between mental disorders and the use of alcohol, tobacco and illicit drugs. Drug and Alcohol Review, 25, 515-36. Recuperado de: http://www.tandfonline.com/doi/ full/10.1080/09595230600944461?scroll=top\&needAccess=true.

Kosttelecky, K. L. (2005). Parental attachment, academic achievement, life events and their relationship to alcohol and drug use during adolescence. Journal of adolescence, 28, 665-669. Doi:10.1016/j.adolescence.2004.12.006.

Le Breton, D. (2003). Adolescencia bajo riesgo. Montevideo, Uruguay: Ediciones Trilce.

Molinatti, F., \& Peláez, E. (2012). Los patrones espaciales de los comportamientos de riesgo en la ciudad de Córdoba (Argentina)-2001. Revista brasileira de estudos populacao, 29(1), 37-52. Recuperado de: http://www.scielo.br/pdf/rbepop/v29n1/v29n1a03.pdf.

O'donogue, T. y Rabin, M. (2000). En S. Cueto, V. Saldarriaga y I. Muñoz (2011). Conductas de riesgo entre adolescentes peruanos: un enfoque longitudinal. Consejo Latinoamericano de Ciencias Sociales (CLASCO) (Ed.) (pp. 118-165). Lima, Perú: GRADE. Recuperado de: http:// repositorio.grade.org.pe/bitstream/handle/GRADE/126/30_cueto_saldarriaga_munoz. pdf? sequence $=1$ \&isAllowed $=\mathrm{y}$.

Oliva, A. (2004). La adolescencia como riesgo y oportunidad. Infancia y Aprendizaje, 27(1), 115-122. https://personal.us.es/oliva/p115.pdf.

Orcasita Pineda, L. y Uribe Rodríguez, A. (2012). La importancia del apoyo social en el bienestar de los adolescentes. Psychologia: avances de la disciplina, 4(2), 69-82. Recuperado de: http://www. scielo.org.co/pdf/psych/v4n2/v4n2a07.pdf.

Paniagua Repetto, H. y García Calatayud, S. (2003). Signos de alerta de trastornos alimentarios, depresivos, del aprendizaje y conductas violentas entre adolescentes de Cantabria. Revista Española de Salud Pública, 77, 411-422. Recuperado de: http://scielo.isciii.es/pdf/resp/v77n3/ original8.pdf.

Páramo D. L. Á. (2011). Factores de Riesgo y Factores de Protección en la Adolescencia: Análisis de Contenido a través de Grupos de Discusión. Terapia psicológica, 29(1), 85-95. http://dx.doi. org/10.4067/S0718-48082011000100009. 
Pineda Pérez, S. y Aliño Santiago, M. (1999). El concepto de adolescencia En Ministerio de Salud Pública de Cuba. Manual de prácticas clínicas para la atención integral a la salud de la adolescencia, pp. 1-9. La Habana, Cuba: MINSAP. Recuperado de: http://ccp.ucr.ac.cr/bvp/ pdf/adolescencia/Capitulo\%20l.pdf.

Restrepo Henao, A. R., Duque Ramírez, L. F. y Montoya Gómez, N. E. (2012). Programa de prevención temprana de conductas de riesgo para la salud en preescolares y escolares. Medellín, 2009-2012: una posibilidad para la prevención temprana de las conductas de riesgo en Latinoamérica. Cad. Saúde Colet. Rio de Janeiro, 20(4), 405-415. Recuperado de: http://www. scielo.br/pdf/cadsc/v20n4/v20n4a02.pdf.

Roche, K., Ahmed, S. \& Blum, R. (2008). Enduring consequences of parenting for risk behaviors from adolescence into early adulthood. Social Science \& Medicine, 66(9), 2023-2034. https://doi. org/10.1016/j.socscimed.2008.01.009.

Rosabal García, E., Romero Muñoz, N., Gaquín Ramírez, K., Mérida, H., y Rosa, A. (2015). Conductas de riesgo en los adolescentes. Revista Cubana de Medicina Militar, 44(2), 218-229. Recuperado de: http://scielo.sld.cu/scielo.php?script=sci_arttext\&pid=S0138-65572015000200010.

Sánchez, M. H. (2013). Adolescencia, conductas de riesgo y prevención. En M. H. Sánchez (Ed.), Prevención de lesiones no intencionales: experiencias con adolescentes (pp. 24-34) Cuba: Ediciones Molinos Trade S.A.

Santander, S., Zubarew, T., Santelices, L., Argollo, P., Cerda, J. y Bórquez, M. (2008).

Influencia de la familia como factor protector de conductas de riesgo en escolares chilenos. Revista médica de Chile, 136(3), 317-324. http://dx.doi.org/10.4067/S003498872008000300006.

Vega, A., Aramendi, P. y Garín, S. (2012). Adolescentes y jóvenes: desde las conductas de riesgo a la inclusión social. Zerbitzuan: Gizarte zerbitzuetarako aldizkaria= Revista de servicios sociales, (52), 167-178. Recuperado de: http://www.zerbitzuan.net/documentos/zerbitzuan/ Zerbitzuan52.pdf\#page $=167$.

Velásquez, J. F. (2009). La juventud y la época. Temeridad y cobardía. Patologías de la individualización en el joven contemporáneo. Revista CES Psicología, 2(1), 35-51. ventana, 2(20), 240-266. Recuperado de: http://revistas.ces.edu.co/index.php/psicologia/article/view/352/199. 